\title{
Experimental analysis of the dynamic responses of bridging objects with alternative non-contact method
}

\author{
Boštjan Kovačič ${ }^{1, *}$, Tomaž Motoh $^{2}$, and Samo Lubej ${ }^{1}$ \\ ${ }^{1}$ University of Maribor, Faculty of Civil Engineering, Transportation Engineering and Architecture, \\ Smetanova ul. 17, 2000 Maribor, Slovenia \\ ${ }^{2} \mathrm{Q}$ Techna, Institute of Quality Assurance and Quality Control Ltd. Ljubljana, Slovenia
}

\begin{abstract}
Structure objects are exposed to different natural influences resulting in long-term and catastrophic outcomes. In order to avoid them, regular monitoring is needed. Various approaches to control condition of a structure can be considered, one of them is the measurement by means of monitoring which can be performed in different ways, depending on responses - static or dynamic, or both simultaneously. Specifically, technological development has enabled to monitor not only static but also dynamic responses by non-contact geodetic methods. Due to their simplicity and reliability of results, geodetic methods are more and more useful in this area. Moreover, state-of-art instruments and additional programme equipment allows up to 100 readings per second with an almost constant frequency of sampling, which is a condition for calculation of a dynamic response according to Fourier's transformation. The article presents noncontact geodetic method of RTS on a bridging object.
\end{abstract}

\section{Introduction}

List of notations (examples below)

RTS $=$ Robotic Total Station

GNSS $=$ Global Navigation Satellite System

GPS $=$ Global Positioning System

FFT $=$ Fast Fourier Transformation

$\mathrm{f}(\mathrm{n})=$ frequency

$\mathrm{Hz}=$ Hertz

$\mathrm{a}=$ amplitude

$\mathrm{k}=$ parameter $\mathrm{kRTS}$

Bridging objects are structures that are exposed to external and internal factors and are therefore considered as critical objects. Their behaviour should be regularly observed in form of control measurement or monitoring which require different methods depending on the level of difficulty. Measurements can be taken with physical or alternative non-contact

\footnotetext{
*Correxponding author: bostjan.kovacic@um.si
} 
methods, above all geodetic (optical) methods. Methodical monitoring in the phase of exploitation of the object, project calculations can be confirmed, safety and reliability are checked. For this purpose, static and dynamic load tests are carried out separately, whereas control and deformation geodetic measurements are performed in the form of daily, monthly and annual geodetic monitoring. The control and deformation geodetic measurements differ in the focus of control geodetic measurements which are on the absolute value of observed points, while deformation measurements concentrate on change of position (displacements) of observed points (Ogundare 2016).

In practice, displacements are measured only in single points with a limited range of instruments demanding lots of time and means which usually cause problems in structure measuring and testing. The result of a non-contact measurement system are displacements in individual points on different places. In addition, optical systems are more accurate, measurements are more transparent, preparation for measurements is not necessary anymore. Automatic point acquisition enables a quick and reliable determination of point positions.

Fast technological development and constant improvement of geodetic instruments and measurement methods allow for performance of more complex and demanding tasks also for engineering geodesy. Static tests are performed with classic geodetic instruments and methods such as levelling, trigonometric altimeter, photogrammetry and terrain scanning. Modern geodetic instruments enable measurement of dynamic responses because they can take up to 100 readings per second. Moreover, the reconstruction of oscillation signals is subjected to the sampling frequency, which means that increasing the number of readings per second results in better accuracy.

The authors (Celebi and Sanli 2002, Chen, Huang, Ding, Xu and Ko 2001, Roberts, Meng, Dodson 2001, Roberts, Cosser, Meng and Dodson 2004, Ogaja, Wang and Rizos 2003, Meng, Dodson and Roberts 2007, Marendić, Paar, Duvnjak and Buterin 2013) deal with data acquisition in dynamic responses with GNSS methods which are the simplest to use. They eliminate the deficiencies of the GNSS methods, such as irregular sampling after elimination of low-quality and false measurements (multipath, poor satellite arrangement...), the unreliability of height component or signal disturbance, by introducing additional geodetic measurements with RTS robotic total station which automatically gathers position data. Various authors (Stempfhuber 2000, Radovanović and Teskey 2001, Kopačik, Kyronović, Kadlecikova 2005, Ceryova, Kubanka, Kopačik, Kyrinović 2002, Chua 2004, Cosser, Roberts, Dodson, Meng 2003, Gikas and Daskalakis, 2006) discuss synchronization of the RTS measurement signal which proved to be irregular for research on dynamic responds of a structure. This irregularity was eliminated by the development of technology and additional programmes. Lekidis, Tsakiri, Makra, Karakostas, Klimis and Sous (2005), Jauregui, White, Woodward and Leitch (2002) show that the best way of measuring is combining various methods (GPS, RTS, Photogrammetric and accelerometers) and comparing results whenever structure, conditions and time allow this.

Nowadays, the RTS method enables up to 30 measurements per second which suffice the monitoring of slow and steady dynamic responses. By this, we mean flexible bridging objects which are subject to oscillations of some centimetres. The measurements can be taken by physical methods, which are more reliable, or by geodetic ones, which are simpler. Certainly, the usage of both methods simultaneously is reasonable. However, some objects do not allow installation of physical methods due to their specificity, so we are forced to find an alternative method which would enable reliable results for further analysis of dynamic responses (Xuan, Cai, Lu and Zhang 2017). Previous research proves that one of the best alternative methods is the RTS non-contact geodetic method.

\section{Description of the object and the measurement equipment}


Railway bridge across the Mura River is a part of railway line No 41 Ormož - Hodoš. The design of the bridge includes three equal simply supported steel truss structures with polygonal top chord and spans between supports $3 \times 51.8 \mathrm{~m}$. It also consists of two bridge abutments and two river piles, as seen from Fig 1. The rail across the bridge runs straight. The $5 \mathrm{~m}$ wide single-track bridge was built in 1953.

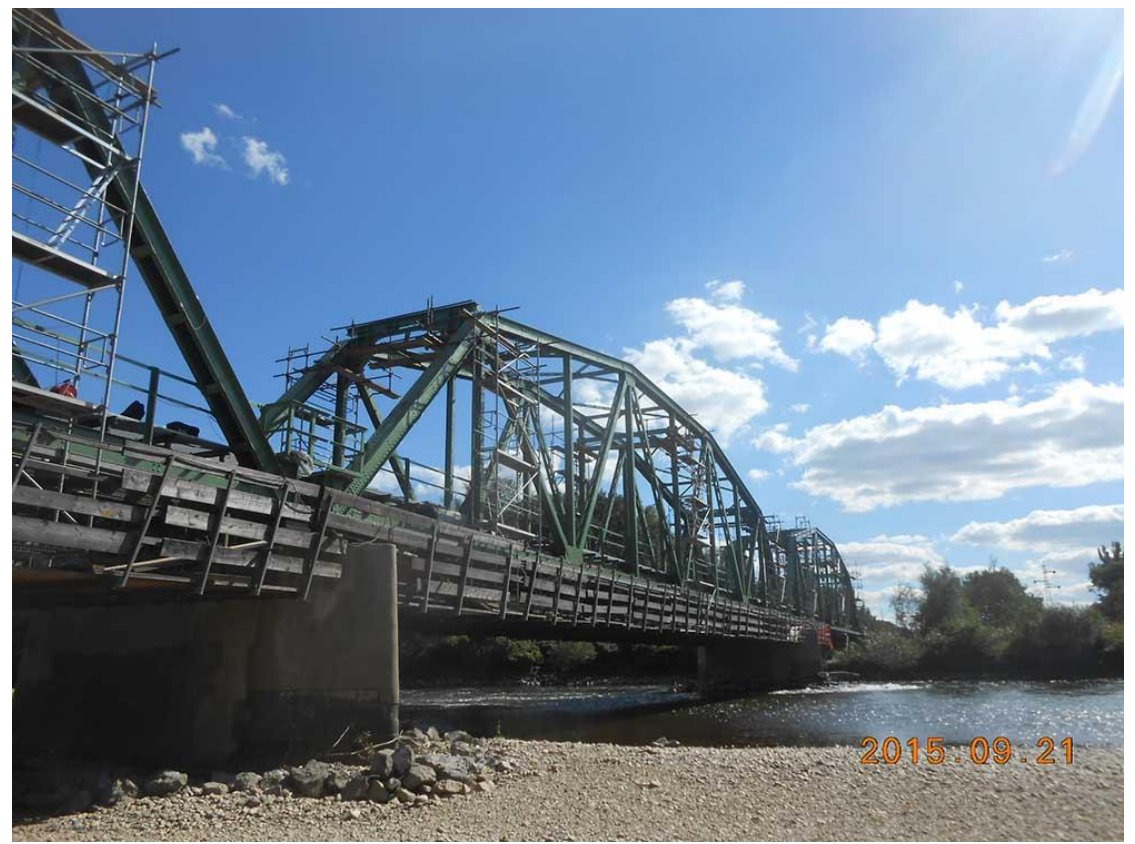

Fig 1. Railway bridge during reconstruction (Source: IMK Institute for metal structures).

The measurements of dynamic characteristics of the railway bridge were taken in the middle of June in 2017. Besides data on bridge response to the dynamic load of rolling stock, the position of geodetic prisms attached to the bridge was recorded after each action. The standing point of the robotic total station Leica TS 50 was next to the bridge as shown in Fig 2 and enabled optimal vision to four prisms (two Pentagon prisms and two reflex selfadhesive prisms). The responses of the structure on the dynamic load of rolling stock were monitored by prisms which were attached to the vertical chord of the truss in the middle of the field span. The aim of the measurements was to acquire data on the dynamic characteristics of the bridge after reconstruction. The stirring of the structure was caused by two railway compositions. 


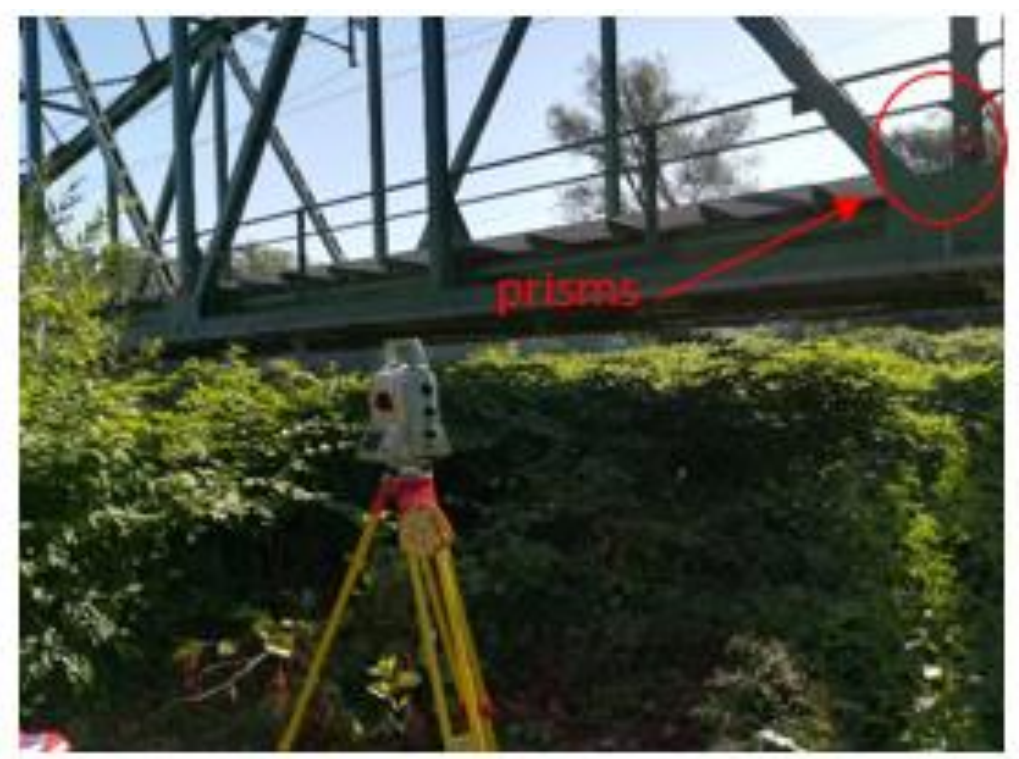

Fig 2. Used geodetic equipment Leica TS 50 and prisms.

\subsection{Processing of acquired results}

Measurements of oscillations and prism positions were taken in four situations which were the basis for analysis of dynamic structural characteristics.

- Determination of ambient oscillation.

- $\quad$ Situation 1 (train crossing 1).

- Situation 2 (train crossing 2).

- Condition after train crossing.

The first phase should show the level of ambient oscillation leading to the findings whether later structure responses to dynamic load are intensive enough to reach the noise threshold. The level of ambient oscillation is determined for the vertical and horizontal direction. Data about changes of vertical and horizontal angle and known coordinates for the position of the instrument and the prism enable calculation of corresponding oscillation with the time-dependent deviation of the observed point from the standstill position. The noise threshold or ambient oscillation of the bridging construction in each direction can be seen in Fig. 3. 


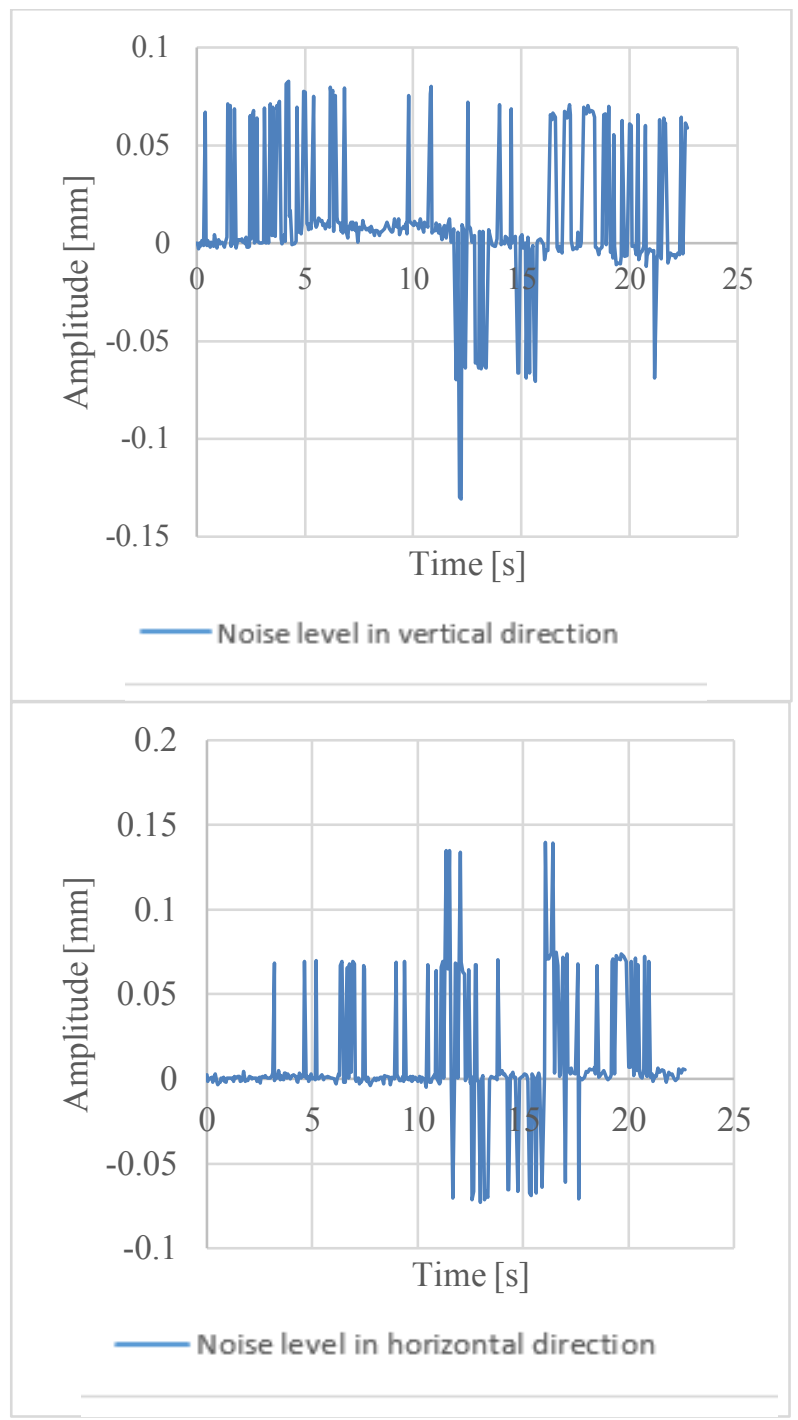

Fig. 3. Ambient oscillation of the railway bridge.

Fig. 3 provides the level of the ambient oscillation. The amplitude of the ambient oscillation ranges mostly around $\pm 0.07 \mathrm{~mm}$ in both vertical and horizontal direction; however, some isolated amplitudes range up to $\pm 0.14 \mathrm{~mm}$. The recorded values of the amplitudes of ambient oscillation are in the case of the used method rather low, therefore, the noise threshold is determined as $\pm 0.14 \mathrm{~mm}$ so that higher reliability is ensured.

Situation 1 presents the measurements of oscillation of the bridging construction caused by the first train. Only responses in the vertical direction are recorded with the aim to acquire quality data under the assumption that the rail runs in a straight line over the bridge, resulting in a smaller lateral impact on construction.

The accurate interval of the train crossing the bridge should be determined in the first step from the footage because the responses appear before and after the train crossing. The robotic total station automatically records space data about the position of a prism with its own time search and the unit millisecond. Then the time axle is relatively presented with the starting point zero. The next step is the presentation of oscillation with the high-frequency component 
which is more suitable to determine desired parameters of responses. Graphs 2 and 3 show the original recording of oscillation and the high-frequency component of original oscillation with the marked interval of train crossing in relative time (Situation 1).

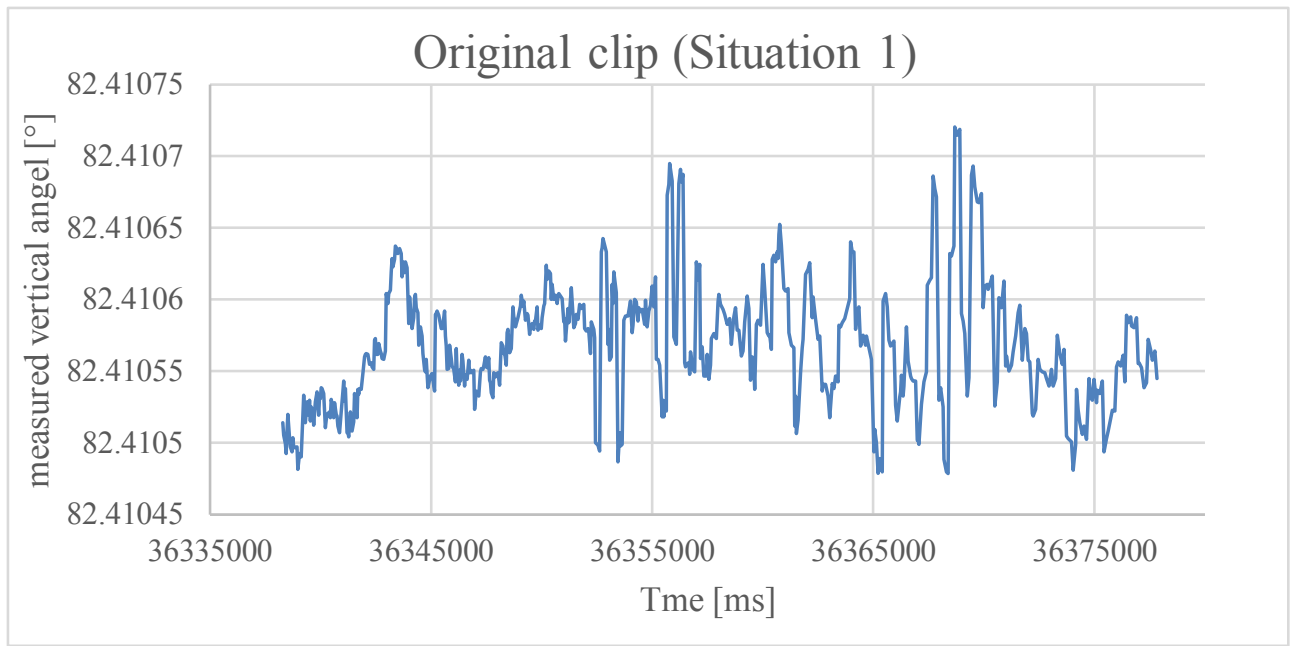

Fig. 4. The original recording of a train crossing the bridge (Situation 1).

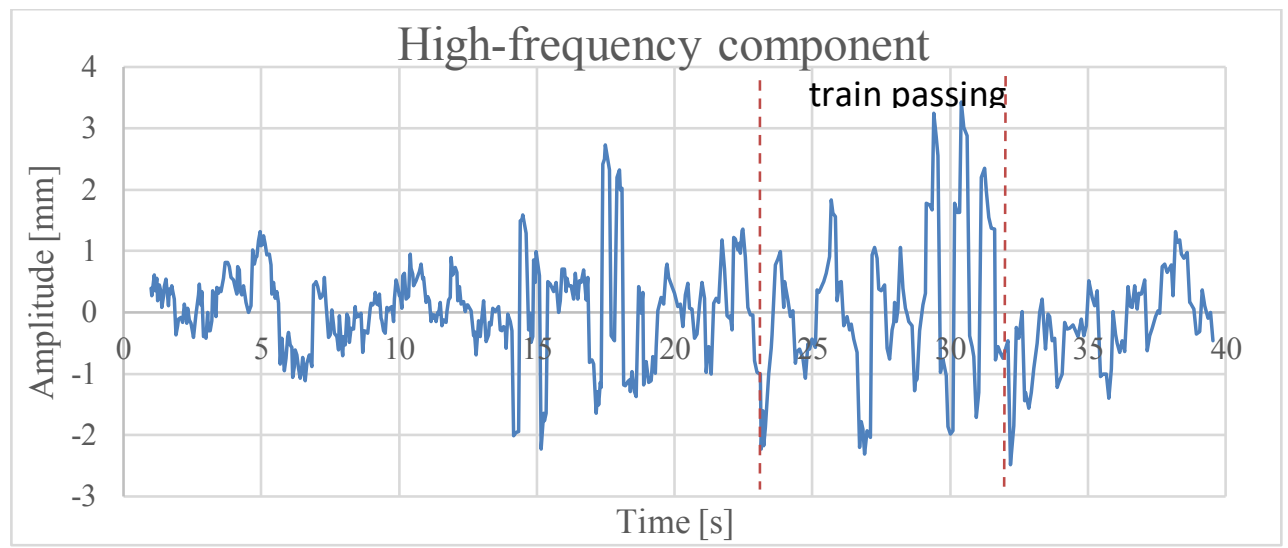

Fig. 5. High-frequency component of oscillation with a marked interval of train crossing (Situation 1).

The relatively short length of the train results in the short time interval of a train crossing the bridge. The highest amplitudes of oscillation are noticed in the time of direct train load on the bridging structure. In addition, a considerably active activity can be observed before direct train load and a bit smaller after the direct load. Spectre analysis is therefore performed for two time intervals. The first interval is determined according to oscillation intensity $(17 \mathrm{~s}$ $-33 \mathrm{~s})$, whereas the second is determined for the interval of the train crossing only (23s 32s). The spectre analysis is performed with two methods (Quick Fourier transform and Lomb-Scargle periodogram) in order to compare results as shown in Fig 3. 


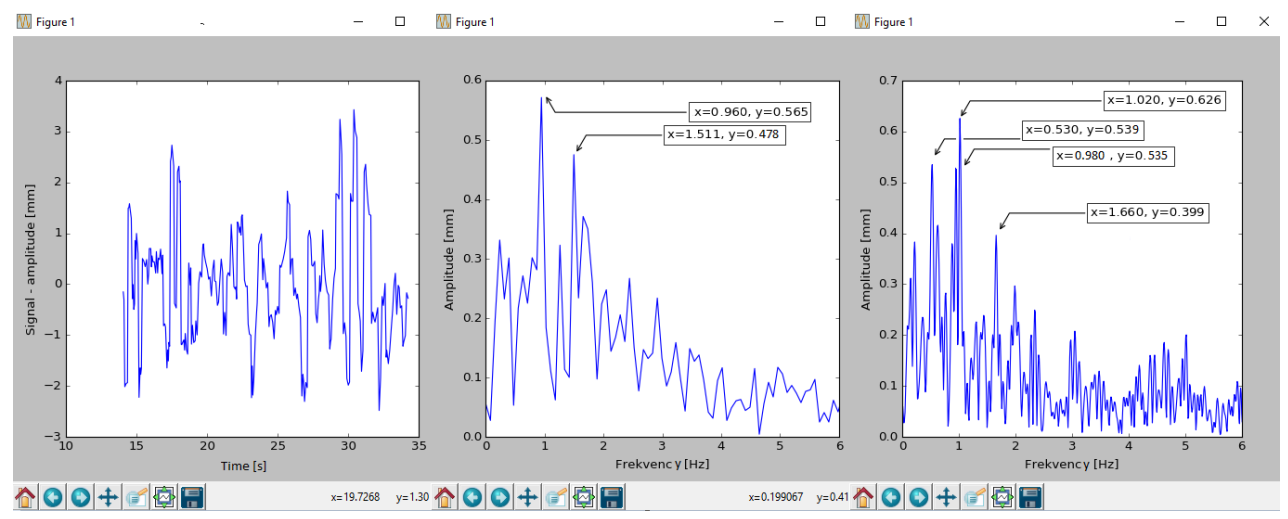

Fig. 6. Spectre analysis for the first interval: left (signal), middle (FFT), right (Lomb-Scargle).

According to the diagrams in Fig 6, it can be concluded that both methods offer values of typical frequencies and corresponding amplitudes. Even though both methods show similar results, we think that results from Lomb-Scargle are more suitable if we consider the fact that sampling was not completely steady. Due to the algorithm in the FFT method, the number of input data is limited; 256 signal values were chosen for the first signal. Extremes in Fig 6 are marked with coordinates $\mathrm{x}$ (frequency) and $\mathrm{y}$ (amplitude). This analysis studies the time interval which includes responses before and after the direct bridge load. Afterwards, the frequency analysis of responses is performed for the interval where the impact of the dynamic load is direct as shown in Fig 7. 128 signal points are chosen for the FFT method analysis.

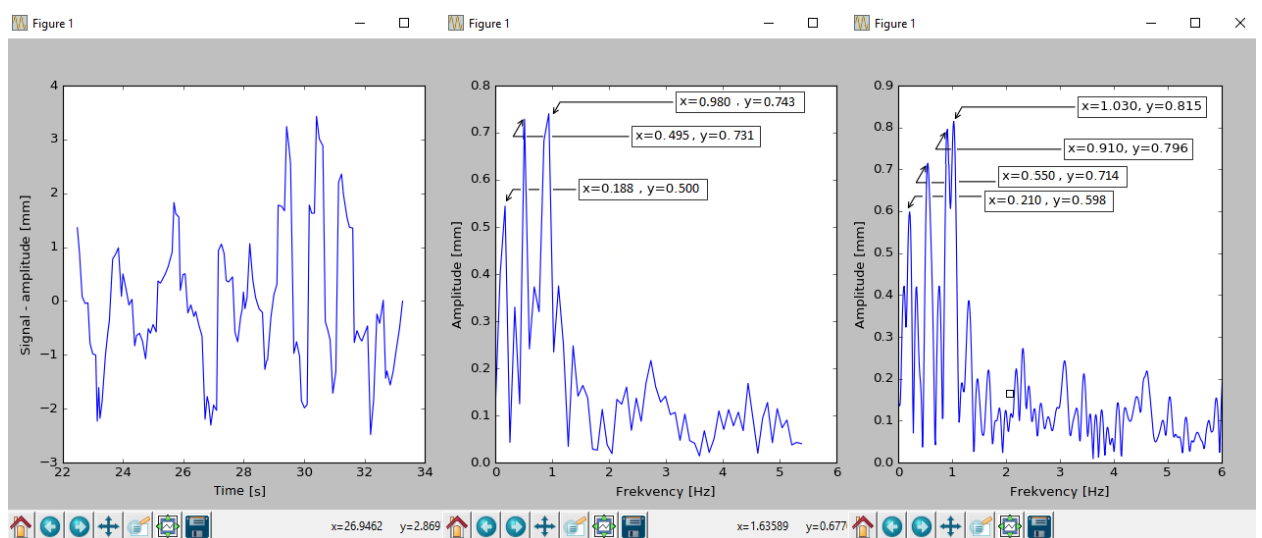

Fig. 7. Spectre analysis for the second interval: left (signal), middle (FFT), right (Lomb-Scargle).

The comparison of frequency spectres of the Lomb-Scargle method in both time intervals shows considerable coincidence of typical frequencies. The comparison of typical frequencies can be found in Table 1.

Table 1. Comparison of typical frequencies.

\begin{tabular}{|l|l|l|}
\hline \multirow{2}{*}{} & \multicolumn{2}{|l|}{ Lomb-Scargle periodogram } \\
\cline { 2 - 3 } & First Interval & Second Interval \\
\hline f1 & $1,02 \mathrm{~Hz}$ & $1.03 \mathrm{~Hz}$ \\
\hline f2 & $0,98 \mathrm{~Hz}$ & $0.91 \mathrm{~Hz}$ \\
\hline f3 & $0.53 \mathrm{~Hz}$ & $0.55 \mathrm{~Hz}$ \\
\hline f4 & $1.66 \mathrm{~Hz}$ & $/$ \\
\hline f5 & $/$ & $0.21 \mathrm{~Hz}$ \\
\hline
\end{tabular}

Note: $\mathrm{fn}=$ frequency. 
The result leads to the conclusion that the dynamic train load impacts the structure in the observed point also before the direct load. Above all, the responses in the second time interval in the direct bridge load are interesting for us while the results of the spectre analysis from the first-time interval can be helpful in interpreting of the origin of each typical frequency. The analysis results show that higher frequencies and frequencies which perfectly correspond in both intervals the structural natural frequency. The frequency $0.21 \mathrm{~Hz}$, which is more intensive in the second interval, is the imposed oscillation frequency.

The middle oscillation amplitude is determined for the treated signal in the second interval according to the procedure, described by Psimoulis and Stiros (2012). The results are displayed in Table 2 .

Table 2. The solution of the numerical procedure for the determination of the oscillation amplitude.

\begin{tabular}{|l|l|l|l|l|l|l|}
\hline$\lambda$ & $k^{\mp}$ & $\varphi^{+}[\mathrm{mm}]$ & $\widehat{a}^{+}[\mathrm{mm}]$ & $\Delta \lambda^{+}[\mathrm{mm}]$ & $\widehat{a}^{-}[\mathrm{mm}]$ & $\Delta \lambda^{-}[\mathrm{mm}]$ \\
\hline 1 & 0.34 & 0.446142 & 1.560232 & 1.839768 & -1.19735 & -2.20265 \\
\hline 2 & 0.68 & 0.892284 & 1.884139 & 1.515861 & -1.59943 & -1.80057 \\
\hline 3 & 1.02 & 1.338427 & 2.096977 & 1.303023 & -1.96577 & -1.43423 \\
\hline 4 & 1.36 & 1.784569 & 2.633651 & 0.766349 & -2.03858 & -1.36142 \\
\hline 5 & 1.7 & 2.230711 & 2.902315 & 0.497685 & -2.26885 & -1.13115 \\
\hline 6 & 2.04 & 2.676853 & 3.082577 & 0.317423 & $/$ & $/$ \\
\hline 7 & 2.38 & 3.122995 & 3.334699 & 0.065301 & $/$ & $/$ \\
\hline 8 & 2.72 & 3.569137 & $/$ & $/$ & $/$ & $/$ \\
\hline 9 & 3.06 & 4.01528 & $/$ & $/$ & $/$ & $/$ \\
\hline 10 & 3.4 & 4.461422 & $/$ & $/$ & $/$ & $/$ \\
\hline
\end{tabular}

Note: $\lambda=\operatorname{step}, k=$ parameter for RTS, calculated filter, $a=$ amplitude, amplitude deviation.

Table 2 shows substantially accurate determination of oscillation amplitude for the marked time interval from Fig. 5. The algorithm in our case, where oscillation does not have harmonic form, leads to the estimation of the highest amplitude in both directions. The algorithm in this example does not show any unknown amplitude, because the highest amplitude value can be taken from the signal diagram.

Already the fifth step leads to the negative amplitude value and the seventh to the positive amplitude value. The difference between middle amplitudes in both directions is considerably high. The reason for that lies in the nature of studied oscillation because it is composed of many frequencies with different amplitudes, which include the recorded signal. All these single frequencies with corresponding amplitudes are displayed on the signal spectrogram for the Interval 1, shown in Fig 5. Amplitudes in positive direction increase imposed oscillation.

Situation 2 shows the response of the structure to the trainload with three segments. The responses of the structure were measured in the horizontal and vertical direction in the same point as in Situation 1 so that the largest response appears at the beginning and it then decreases. 

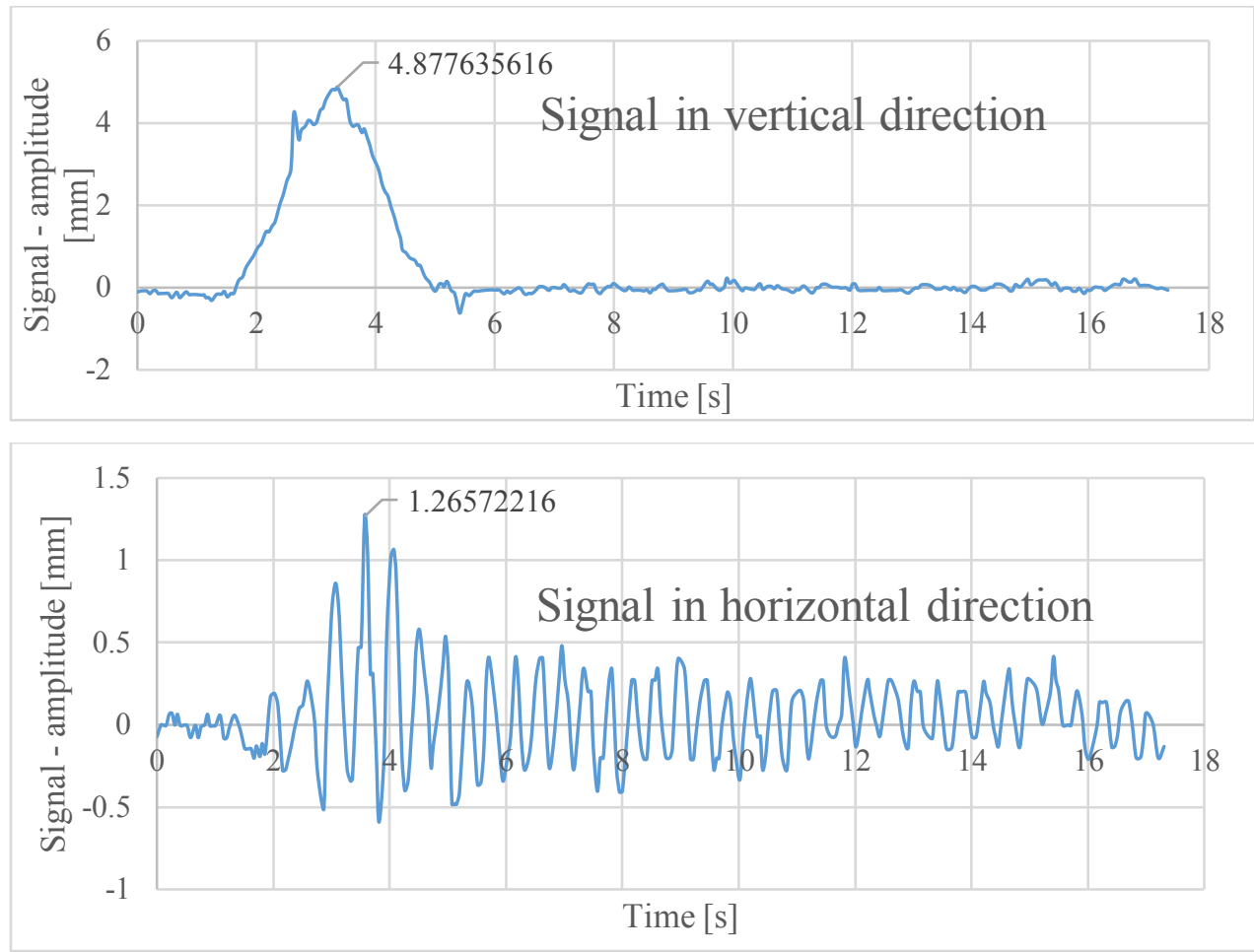

Fig. 8. The signal in the vertical and horizontal direction for the steel bridge in Situation 2.

The diagram in Fig. 8 shows a signal in the vertical and horizontal direction at a train load on a railway bridge with three units. The signal in the vertical direction clearly displays the time interval in which the train crosses the bridge. In this case, the signal almost lacks oscillation, or the oscillation amplitude is very low. The form of response resembles apparent static activity, while responses in horizontal direction show clear oscillation which is in the phase of direct load the most intensive.

Regarding the form of responses of the structure in each direction, the spectre analysis can only be performed on the signal describing the oscillation in the horizontal direction. The results of the previous activity show that analysing more time intervals of the sample signal is sensible. Therefore, the time interval at the direct load of the measurement point is chosen, which starts at the approximate time $1.8 \mathrm{~s}$ and ends at the time of $5 \mathrm{~s}$. In order to compare frequency spectre, the random signal from $5 \mathrm{~s}$ to $10 \mathrm{~s}$ is selected for the second interval according to the response size, which can be seen in Fig 5. We can expect that certain main frequencies will be similar at both intervals; the difference will be in intensity. The frequency analysis of the sample signal is performed with the Lomb-Scargle method, in programme language Python. 


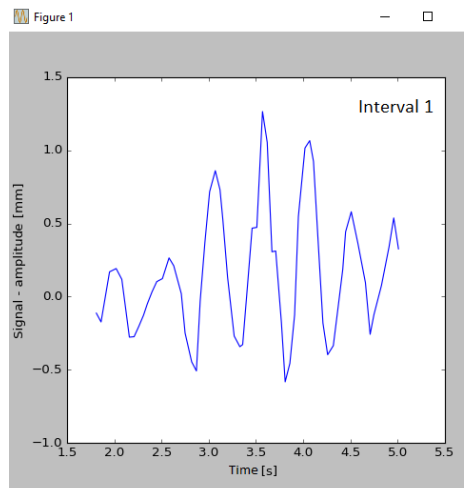

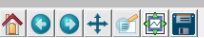

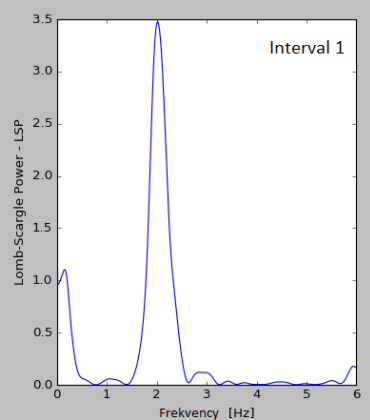

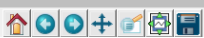

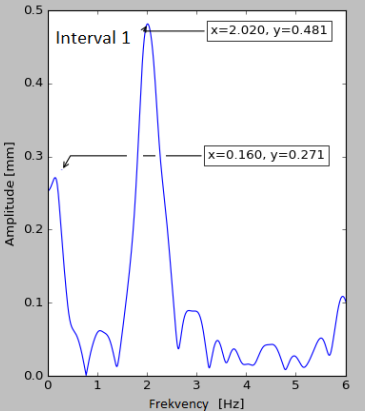

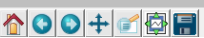

Fig. 9. Spectre analysis, the second activity, Interval 1: left (signal), middle (intensity Lomb-Scargle), right (normalized Lomb-Scargle periodogram).

Fig. 9 shows the main frequency $2.02 \mathrm{~Hz}$ and the frequency $0.16 \mathrm{~Hz}$. The frequency $2.02 \mathrm{~Hz}$ corresponds to one of the structural own frequency, whereas the frequency $0.16 \mathrm{~Hz}$ is regarded as the imposed train frequency. The spectres from the first and the second activity are different which is not what we expected. However, two different activities with different loads can suggest that the structure responses completely otherwise and oscillate with different frequency. The same procedure is used for the time interval 2, as shown in Fig. 10.

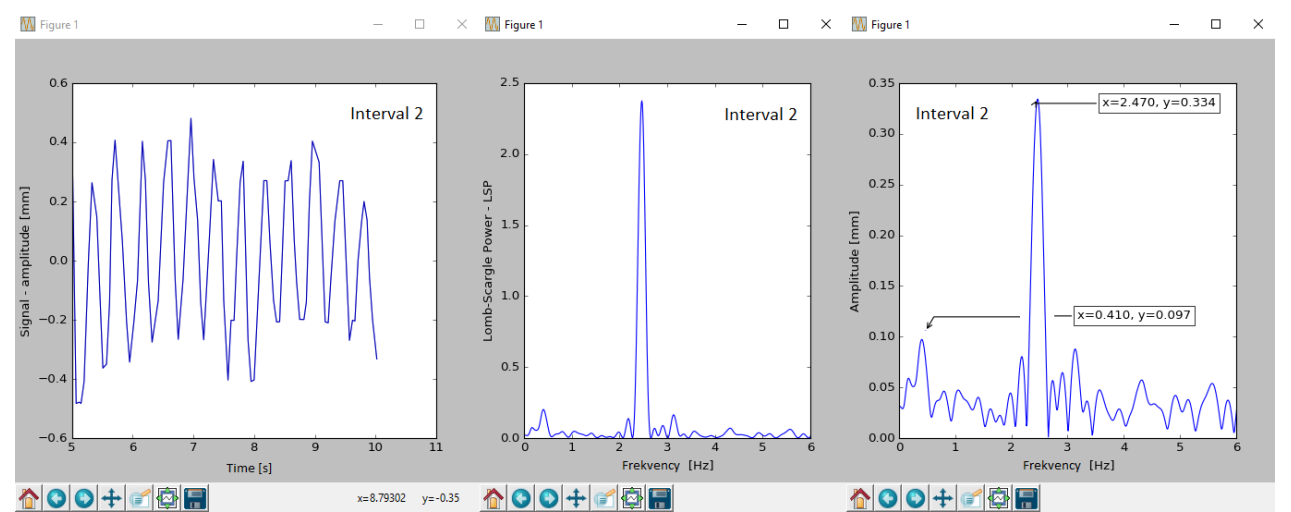

Fig. 10. Spectre analysis for the second activity, Interval 2: left (signal), middle (intensity LombScargle), right (normalized Lomb-Scargle periodogram).

The results of the second analysis show an even oscillation of the structure in the horizontal direction after the direct load with the main frequency $2.47 \mathrm{~Hz}$ which presents one of the structural own frequencies. The frequency resembles the main frequency from the first interval, but it does not coincide, which does not meet our expectations. In addition, the frequency $0.41 \mathrm{~Hz}$ appears with lower intensity and represents one of the structural own frequencies.

Fig 10 (right) shows a relatively low noise level. If the middle amplitude is evaluated to $0.4 \mathrm{~mm}$ and the main frequency presents oscillation with the amplitude $0.334 \mathrm{~mm}$, the level of importance of the main frequency can be determined by the quotient of these two values of frequencies. The level of importance of the main frequency equals $0.334 / 0.4$ or 0.835 . 


\section{Discussion}

The results of measurements of dynamic responses show the progress of geodetic methods in the establishment of structure condition. However, the problem of sampling is still present which limit the precision of geodetic methods. As a result, measurements of geodetic methods cannot yet reach the same accuracy as other physical methods for the determination of dynamic structural characteristics which means that both should be combined in order to get precise analyses. Non-contact geodetic methods are a fast, accessible and useful tool in construction, which provides enough accuracy to determine dynamic characteristics of objects during construction and periodical monitoring of structure condition in the time of their usage.

\section{Conclusion}

The results of our experiment and the results of previous research confirm the usefulness of the non-contact geodetic method in determination of the dynamic responses of structures. Our case study includes the spectrum of frequencies from $1 \mathrm{~Hz}$ up to approximately $5 \mathrm{~Hz}$ and amplitudes from $0.1 \mathrm{~mm}$ to $5 \mathrm{~mm}$ which proved to be the most appropriate in these ranges of frequencies according to earlier research.

The results of measurements on the railway bridge reveal a quite rigid structure. The biggest vertical displacement $3.4 \mathrm{~mm}$ was noted in the first situation. The spectre analysis showed a rather uneven signal with numerous frequencies, and the main among them was approximately $1 \mathrm{~Hz}$. In the second situation, a curve without oscillation appeared in the vertical direction. The largest displacement, in this case, was almost $4.9 \mathrm{~mm}$. In the horizontal direction, the oscillation with the largest amplitude $1.26 \mathrm{~mm}$ and the main amplitude $2 \mathrm{~Hz}$ was recorded. Since the bridge was loaded by two lighter and relatively short trains, it would be sensible to repeat the measurements at the crossing of a long freight train with larger mass. In such case, the signal would be more intense and longer and consequently more appropriate for the analysis.

The signal analysis with methods FFT and Lomb-Scargle proved to be an excellent tool to present data on frequency oscillation, even though the FFT method does not consider the uneven sampling of a signal. The method was suitable for the determination of frequency spectre in sufficient accuracy. FFT method seems to be more useful, but it could be performed only to even sampling of the RTS system. However, better accuracy could be reached by the Lomb-Scargle method.

In conclusion, the geodetic non-contact method for monitoring of object responses seems to best meet the requirements for the continuing observation of an object under various influences and factors in the form of 24/365 monitoring, where other methods do not give all necessary results. The method is, therefore, suitable for subsequent static and dynamic calculations of construction of buildings and other structures.

\section{References}

1. J.O. Ogundare, Precise surveying: The Principles and Geomatics Practise (Wiley, New Jersey, USA, 2016)

2. M. Celebi, A. Sanli, Earthq. Spec. 18(1), 47-61 (2002)

3. Q. Chen, D.F. Huang, X.L. Ding, Y.L. Xu, J.M. Ko, Proceedings of health monitoring and management of civil infrastructure systems (Bellingham (WA), SPIE, 2001) 
4. G.W. Roberts, X. Meng, A.H. Dodson, Proc. 10th FIG Int. Symp. on Deformation Measurements, 19-22 (2001)

5. G.W. Roberts, E. Cosser, X. Meng, A.H. Dodson, Proc. 17th International Technical Meeting of the Satellite Division of the U.S. (Institute of Navigation, Long Beach, California, 2004)

6. C. Ogaja, J. Wang, C. Rizos, Journal of Surveying Engineering 129(3), 99-104 (2003)

7. X. Meng, A.H. Dodson, G.W. Roberts, Engineering Structures 29, 3178-3184 (2007)

8. A. Marendić, R. Paar, I. Duvnjak, A. Buterin, Proc. 6th International Conference on Engineering Surveying (Praga, 2014)

9. P.A. Psimoulis, C.S. Stathis, Journal of Bridge Engineering 18(2) (2013)

10. W. Stempfhuber, Proc. 3rd International Symposium on Mobile Mapping Technology (Cairo, 2000)

11. W. Stempfhuber, Optical 3-D Measurement Techniques IX (Vienna, 2009)

12. R.S. Radovanovic, W.F. Teskey, Proc., 10th FIG symposium on deformation measurements (Orange, California, 2001)

13. A. Kopačik, P. Kyronović, V. Kadlecikova, Proc. FIG working week, From Pharaohs to Geoinformatics TS 22.9 (2005)

14. I. Ceryova, P. Kubanka, A. Kopačik, P. Kyrinović, Proc. FIF XXII International Congress (Washington, D.C. USA, 2002)

15. E. Cosser, G.W. Roberts, A.H. Dodson, X. Meng, Journal of Applied Geodesy 1(3), 171179 (2003)

16. S.C. Chua, Testing of Robotic Total Stations for Dynamic Tracking, Dissertation (The University of Southern Queensland, Australia, 2004)

17. V. Lekidis, M. Tsakiri, K. Makra, C. Karakostas, N. Klimis, I. Sous, Eng Geol. 79(1-2), 43-59 (2005)

18. D.V. Jauregui, R.K. White, C.B. Woodward, K.R. Leitch, Journal of Bridge Engineering 8, 212-222 (2003)

19. K. Xuan, C.S. Cai, D. Lu, W. Zhang, Journal of Bridge Engineering 22(6) (2017)

20. P.A. Psimoulis, S.C. Stiros, Computers and Structures 92-93, 337-348 (2012) 